\title{
A INTRODUÇÃO DA CONCEPÇÃO DE EXCELÊNCIA GERENCIAL NOS INSTITUTOS E CENTROS DE PESQUISA BRASILEIROS - O PROJETO EXCELÊNCIA NA PESQUISA TECNOLÓGICA
}

\author{
Marconi Edson Esmeraldo Albuquerque \\ medson@cnpq.br \\ Conselho Nacional de Desenvolvimento Científico e Tecnológico - Brasília, DF/Brasil \\ Maria Beatriz Machado Bonacelli \\ bia@ige.unicamp.br \\ Universidade Estadual de Campinas - São Paulo, SP/Brasil
}

Recebido em 08/07/2009

Aprovado em 22/09/2010

Disponibilizado em 01/12/2011

Avaliado pelo sistema double blind review

Revista Eletrônica de Administração

Editor: Luís Felipe Nascimento

ISSN 1413-2311 (versão on-line)

Editada pela Escola de Administração da Universidade Federal do Rio Grande do Sul.

Periodicidade: Quadrimestral

Sistema requerido: Adobe Acrobat Reader.

\section{RESUMO}

Programas de excelência em desempenho organizacional têm se proliferado mundo afora, transbordando sua filosofia e diretivas para além da indústria. O interesse de diferentes organizações por processos de avaliação se deve à evolução dos modelos de excelência gerencial (MEGs) americano e europeu. No Brasil, o MEG do Prêmio Nacional da Qualidade (PNQ) vem se propagando como referência para a constituição de programas de premiação em várias áreas. Os institutos e centros de pesquisa (ICPs) brasileiros, destacadamente os de natureza pública, têm enfrentado nas últimas décadas uma forte pressão em relação à necessidade de gerarem recursos que garantam sua manutenção e sobrevivência. Isso tem exigido dessas organizações um reposicionamento estratégico, de forma a obter vantagens a partir das interações que estabelecem com os demais atores do sistema de inovação. Dessas instituições têm sido demandadas uma maior capacidade de resposta e a formulação de suas ações com base em diretrizes estratégicas consistentes. Evidentemente, os mecanismos de gestão passam a ter uma importância singular para que se alcancem os objetivos traçados. Este artigo tem por objetivo analisar o movimento pela excelência gerencial nos ICPs brasileiros, capitaneado pela Associação Brasileira das Instituições de Pesquisa Tecnológica (ABIPTI) e materializado no Projeto Excelência na Pesquisa Tecnológica (PEPT). Para tanto, buscam-se referenciais: i) que permitam a descrição e análise da evolução da qualidade na sociedade contemporânea, tendo como marcos os modelos de produção americano e o japonês; ii) que possibilitem a compreensão da criação e desenvolvimento dos principais prêmios de qualidade, inclusive o brasileiro; e iii) que ajudem a compreender as transformações na área da pesquisa científica e tecnológica. Além de revisão bibliográfica 
Marconi Edson Esmeraldo Albuquerque \& Maria Beatriz Machado Bonacelli

sobre o assunto, foram examinados documentos referentes ao PEPT, o que possibilitou uma melhor compreensão do contexto de sua criação, o papel da ABIPTI nesse processo e a escolha do PNQ como modelo a orientar a mudança nos sistemas de gestão dos institutos participantes. Uma das principais conclusões a que chegou esse trabalho é que o PEPT tem tido dificuldades em difundir a 'excelência em gestão' nos ICPs e uma das razões para isso é a dificuldade encontrada pelos mesmos em internalizar processos de gestão em suas estruturas, a exemplo do MEG/PNQ.

Palavras-chave: Evolução da Qualidade na Sociedade Contemporânea; Excelência em Desempenho; Modelos de Excelência Gerencial; Ciência, Tecnologia e Inovação; Institutos de Pesquisa e Desenvolvimento.

\title{
MANAGERIAL EXCELLENCE IN BRAZILIAN RESEARCH INSTITUTES AND CENTERS: THE EXPERIENCE OF 'EXCELLENCE IN TECHNOLOGICAL RESEARCH PROGRAM'
}

\begin{abstract}
Managerial excellence programs have diffused around the world, extending its philosophy and guidelines beyond the industry. The interest of different organizations about organizational assessment processes is because of the development of American and European Business Excellence Models (BEMs). In Brazil, the BEM of the National Quality Award (PNQ) has been spreading as a reference for the establishment of programs for excellence awards in several society areas. In the last decades, Brazilian research and technology organizations (RTOs), especially those of public nature, have faced intense pressure to generate resources to ensure its maintenance and survival. This has required a strategic vision of these organizations, as to obtain benefits from the interactions they establish with other organizations in the innovation systems. RTOs have been compelled to improve their responsiveness and planning their activities on the basis of consistent strategic guidelines. Evidently, the management mechanisms are of great importance to achieve the goals set. This article aims to present the movement for excellence in management of Brazilian RTOs, led by Brazilian Association of Technological Research Institutions (ABIPTI) and embodied in the Excellence in Technological Research Program (PEPT). We have adopted references that: i) enable to describe and analyse of the evolution of Quality in contemporary society, taking as benchmarks the American and Japanese production archetypes, ii) enable to understand the creation and development of major quality awards, including the Brazilian, and iii) help to perceive the changes in the scientific and technological research. Besides the literature review, we examined some documents relating to PEPT Program, which allowed a better understanding of the context of its creation, the role of ABIPTI in this process and the choice of PNQ as the model to guide the change in management systems of the associated RTOs. One of the main considerations of this paper is that the PEPT Program has had difficulties in spreading the "management excellence" in RTOs and one reason for this is the difficulty encountered by them to internalize management processes in their structures, as BEM/PNQ.
\end{abstract}

Key-words: Evolution of Quality in Contemporary Society; Managerial Excellence; Business Excellence Models; Science, Technology and Innovation; Research and Development Institutes. 
A introdução da concepção de excelência gerencial nos institutos e centros de pesquisa brasileiros - o projeto excelência na pesquisa tecnológica

\section{Introdução}

Institutos e centros de pesquisas (ICPs) são importantes atores nos processos de criação e difusão de conhecimento tecnológico e em muitos países vêm passando por reestruturações na organização de suas atividades e na forma como se relacionam com outros atores do sistema de inovação, reflexo de mudanças na dinâmica global da pesquisa $\&$ desenvolvimento (P\&D) e da inovação. Para lidar com incertezas inerentes a esse contexto, é urgente que esses atores revejam seus sistemas de gestão e construam/revisem capacidades para melhor desempenharem seus papéis e funções.

A evolução da qualidade e sua gestão no século XX e o desenvolvimento paripassu de modelos, ferramentas e práticas têm proliferado, nas últimas décadas, em muitos setores da sociedade. Nos estágios mais recentes desses desenvolvimentos encontram-se os modelos de excelência gerencial (MEGs), que são, em essência, baseados nos preceitos da Gestão pela Qualidade Total (TQM). Tais modelos viabilizaram um pool de premiações em países, regiões e setores econômicos. No caso brasileiro, além de empresas e organizações públicas, vários ICPs (em sua maioria, públicos) vêm adotando MEGs para desenvolver seus sistemas de gestão, como uma estratégia de busca de respostas às transformações no seu ambiente de atuação.

Este artigo discorre sobre o movimento pela excelência gerencial nos ICPs brasileiros, iniciativa esta conduzida pela Associação Brasileira das Instituições de Pesquisa Tecnológica (ABIPTI), no âmbito do Projeto Excelência na Pesquisa Tecnológica (PEPT). Busca-se, assim, resgatar elementos que permitam a compreensão de como este projeto foi criado e vem sendo desenvolvido nos institutos. O recorte metodológico está baseado em revisão bibliográfica sobre os assuntos enfocados, bem como na análise de documentos que evidenciam elementos importantes da trajetória do PEPT e de seu contexto de criação.

O texto está estruturado em quatro seções, além desta que o apresenta. Na primeira buscase compreender como o conceito de qualidade e sua gestão evoluíram no ambiente produtivo, vinculando essa construção aos modelos de produção capitalista vigentes no século XX; a seção 2 apresenta os principais prêmios internacionais da qualidade - Prêmio Deming, Malcolm Baldrige e o Prêmio Europeu, bem como um daqueles que se constituíram no Brasil 
Marconi Edson Esmeraldo Albuquerque \& Maria Beatriz Machado Bonacelli - o Prêmio Nacional da Qualidade (PNQ); na seção 3 discute-se como o MEG do PNQ se difundiu para o ambiente de pesquisa, a partir da narrativa da criação e desenvolvimento do PEPT. Na quarta e última seção são apresentadas as considerações finais do trabalho.

\section{Introdução da noção de qualidade na sociedade contemporânea}

Para entender 'qualidade' e sua evolução no contexto das organizações, faz-se necessário examiná-la à luz da lógica de produção vigente. Nesta seção nos deteremos, pois, a entender como se desenvolveu a função qualidade em relação aos dois sistemas de produção dominantes no século XX - o Sistema de Produção em Massa e o Sistema Toyota de Produção.

Dois autores são importantes na classificação dos momentos que perpassam a evolução da qualidade e sua gestão: David Garvin (1992) e Robert Cole (1999), ambos estudiosos da transformação industrial japonesa. O primeiro autor distingue o desenvolvimento da qualidade em quatro eras: inspeção, controle estatístico da qualidade, garantia da qualidade e gestão da qualidade total, sendo esta última fortemente influenciada pelo aumento no número de processos de indenização em função de produtos, pressões do governo em várias frentes e especialmente a forte penetração de fabricantes japoneses no mercado norteamericano. Para esse autor, as abordagens da qualidade que surgiram ao longo do século XX foram sendo configuradas lentamente e não por meio de inovações marcantes, e todas, em sua sucessão, absorveram elementos das abordagens anteriores.

Já Cole (1999), como ele mesmo afirma, não faz um tratamento histórico da evolução da qualidade, embora demarque o início dos anos 1980 como um marco crítico nessa trajetória ${ }^{1}$. Ele apreende as características analíticas dos elementos que compõem os diferentes avanços na qualidade e as agrupa no que caracterizou de velho e novo modelo da qualidade que, entende-se, estão vinculados, respectivamente, às duas lógicas de produção mencionadas. Além disso, Cole procura incorporar a ênfase da aprendizagem organizacional percebida nos dois modelos. Sem abandonar os argumentos e delimitações de Garvin, as sub-seções seguintes foram construídas apoiando-se na caracterização de Cole.

\subsection{O conceito de qualidade no Sistema de Produção em Massa}

Nos séculos XVIII e XIX, o controle da qualidade de produtos não era uma atividade formalizada no ambiente produtivo. Quase tudo era fabricado por artesãos habilidosos e 
A introdução da concepção de excelência gerencial nos institutos e centros de pesquisa brasileiros - o projeto excelência na pesquisa tecnológica

aprendizes sob a supervisão de mestres de ofício. Os artesãos eram trabalhadores especializados, que dominavam completamente as etapas do ciclo de produção, lidando diretamente com o cliente, interpretando suas necessidades e buscando materializar seus anseios. A percepção da 'qualidade' dos produtos que faziam conferia-lhes reputação perante a sociedade. $\mathrm{O}$ volume de produção era baixo e as peças eram ajustadas manualmente umas às outras. O foco sobre a qualidade se voltava, pois, para o produto, e se viabilizava por meio da inspeção informal (quando feita) de todos os produtos pelo artesão (GARVIN, 1992). Esses são os antecedentes 'produtivos' do que veio depois se conformar como a moderna gestão da qualidade.

A Segunda Revolução Industrial, no início do século XX, trouxe uma nova ordem produtiva, em que a produção artesanal deu lugar à produção em massa de produtos padronizados, possibilitada pela nova forma de organização do trabalho (baseada em intensa divisão do trabalho) e na invenção de máquinas capazes de produzir em grandes quantidades peças intercambiáveis.

O velho modelo de qualidade ergueu-se, pois, no curso da industrialização americana, refletindo especialmente a ascensão da Administração Científica de Taylor, e as contribuições de seus seguidores. Naquele modelo, qualidade era vista como ajustes a padrões, i.e., baseavase na avaliação da conformidade do produto a especificações de projeto. É com Taylor e seu frenético projeto de parcelização do trabalho, que a qualidade é legitimada como uma tarefa distinta das demais no ciclo de produção (GARVIN, 1992). Qualidade tinha, então, uma forte orientação interna, e seus esforços tendiam a se concentrar na área de fabricação. Para Cole (1999), essa ênfase interna levou à negligência de considerações de mercado. Sob esse modelo, a vantagem competitiva buscada pelos gerentes se pautava em elementos como exploração de economias de escala e redução dos custos unitários; qualidade não era pensada como uma área que deveria se preocupar com o consumidor, nem que podia contribuir com a estratégia da empresa.

Assim, qualidade evoluiu como uma função especializada, conduzida por pequenos grupos de especialistas nos departamentos de inspeção/qualidade ${ }^{2}$. Naquele modelo, essas pessoas eram, em geral, os responsáveis pelos resultados da qualidade nas operações de manufatura. Esses profissionais se constituíam, pois, no staff da qualidade nas corporações americanas. A crítica de Cole (1999) é que no modelo americano, o excesso de confiança em 
Marconi Edson Esmeraldo Albuquerque \& Maria Beatriz Machado Bonacelli

especialistas da qualidade levou a uma lamentável abdicação da responsabilidade pela qualidade por muita gente atuando na engenharia e na linha de montagem, e que essa opção implicou que a aprendizagem sobre o controle de qualidade se restringiu a um pequeno grupo de especialistas inaptos. Discorda-se do autor, apenas no uso do termo 'abdicação', que soa como se a não responsabilidade de engenheiros e supervisores de produção pela qualidade na produção se devesse a ato voluntário. Entende-se que isso aconteceu não por renúncia, mas porque eles foram impelidos pela orientação existente naquele modelo.

Como visto anteriormente, nesse modelo o foco do monitoramento de desempenho era via inspeção, e suas atividades contribuíam para manter longe dos clientes os produtos defeituosos. Isso demandava das organizações a estratégia de manter estruturas de serviços para minimizar os efeitos de produtos defeituosos para os clientes. Se isso falhasse, havia serviços pós-vendas para amenizar os efeitos de uma qualidade pobre. Então, mesmo que a inspeção e os serviços para manter aparente a qualidade da produção pudessem contribuir para o alcance de metas da organização, isso ampliava consideravelmente os custos de produção (e, consequentemente, impactava os preços finais dos produtos). Assim, o foco sobre o controle da qualidade no modelo clássico era bastante limitado como meio de controle dos problemas da qualidade e redução da variação de padrões pré-estabelecidos (COLE, 1999).

De uma perspectiva da aprendizagem organizacional, o autor argumenta que essa orientação traz implicações profundas. Para ele, um ciclo repetitivo e um tanto limitado de detecção e recuperação é operacionalizado, exigindo apenas modesta rotação de rotinas organizacionais existentes para se alcançar restritas melhorias. A aprendizagem individual e organizacional para sustentar este ciclo é limitada. Citando Winter (1994), que por sua vez se apoiou na teoria comportamental, o autor conclui que a fase de aprendizado se encerra quando o desempenho é considerado satisfatório (segundo Winter isso também pode acontecer por problemas de falta de atenção). A identificação, eliminação e reparação de defeitos significavam o atingimento dos objetivos da qualidade na lógica clássica de produção, e não era limitado apenas por isso, mas especialmente pela incapacidade de seus idealizadores em compreender as possibilidades da qualidade como objetivo estratégico das organizações.

\subsection{O conceito de qualidade no Sistema Toyota de Produção}

Nos anos 1940 e 1950 especialistas em qualidade americanos - Deming e Juran - já 
A introdução da concepção de excelência gerencial nos institutos e centros de pesquisa brasileiros - o projeto excelência na pesquisa tecnológica

haviam desenvolvido uma abordagem diferente para qualidade, apontando para as vantagens de a fabricação incorporar a preocupação e o controle da qualidade. Contudo, é no Japão que suas contribuições encontram abrigo e terreno fecundo para florescer e, somente depois de sedimentadas naquele país, retornam ao ocidente, já incorporadas na 'lógica japonesa' de produção (ZILBOVICIUS, 1999).

Os elementos que compunham o velho modelo eram, pois, limitados em seu escopo. O novo contexto exigia a rápida redefinição de qualidade nas organizacionais operando sob a lógica tradicional. Era preciso uma visão voltada para fora para se conseguir o comprometimento da alta direção, cujas preocupações precípuas eram estratégia e competição (GARVIN, 1992). A solução foi definir qualidade do ponto de vista do cliente e que estivesse diretamente relacionada a rentabilidade.

Conformidade, embora continuasse sendo importante, não era suficiente para garantir que o desempenho dos produtos atendesse às necessidades do consumidor e passava, então, a ser secundária nessa nova visão. A abordagem voltada para o mercado ampliava o escopo da qualidade, possibilitando a incorporação de novas atividades à função qualidade e fazendo surgir um conjunto de decisões gerenciais diferente daquelas guiadas apenas pelos objetivos de redução de custos, presente na lógica clássica. O foco no mercado implicava que a qualidade passaria a enfatizar as necessidades dos consumidores e que deveriam ser desdobradas amplamente na organização. Isso significa trazer as necessidades do cliente 'para dentro' da organização e sua receptividade a produtos se tornaria a principal referência a orientar novas atividades de melhoria.

Lançando considerações de mercado em suas organizaçãos, gerentes introduzem fontes adicionais de incerteza, além daquelas inerentes aos processos de fabricação. Responder efetivamente a estas pressões demandava motivação, treinamento e flexibilidade através do compromisso de uma força de trabalho descentralizada. Tais fatores garantem mudanças rápidas nas rotinas organizacionais e tanto a aprendizagem individual como a organizacinal fazem parte desse processo (COLE, 1999).

Com a ampliação do escopo da qualidade trazida com o novo modelo, uma linguagem comum de melhoria (contínua) da qualidade se desenvolve em todas as funções e níveis organizacionais, intensificando-se assim, a comunicação, o entendimento e a aceitação de metodologias para solução de problemas. Como frisa Cole (1999), essa uniformidade na 
Marconi Edson Esmeraldo Albuquerque \& Maria Beatriz Machado Bonacelli linguagem não deve ser subestimada, pois ela teve grande projeção sobre o aumento das capacidades de aprendizagem individuais e organizacionais, verificado pelo desenvolvimento de um variado leque de ferramentas utilizados em áreas da organização até então retraídas.

Duas características fundamentais no novo modelo são o enfoque estratégico para a qualidade e a essencialidade do envolvimento de todos (COLE, 1999; GARVIN, 1992). Do desdobramento da primeira surgem incentivos motivacionais para a aprendizagem individual e para que indivíduos incorporem sua expertise nas novas rotinas necessárias em um ambiente de disputa por mercados. Adquire centralidade nesse modelo a indispensável necessidade do envolvimento de todos os empregados e departamentos na melhoria contínua de seus processos de trabalho, visando atender necessidades de seus clientes internos e externos.

Como resumido por Garvin (1992), o novo olhar sobre a qualidade, embora abrangesse aspectos das abordagens que a antecederam, tinha elementos que o distinguiam. Ele era mais amplo, mais intimamente ligado à lucratividade e aos objetivos empresariais básicos, mais sensível à concorrência e ao ponto de vista do consumidor e mais firmemente associado à melhoria contínua. Todavia, Cole (999) se contrapõe à classificação de Garvin das fases da evolução da gestão da qualidade, e da ideia ali implícita de que houve um acúmulo de competências na etapa que enfocou estrategicamente a qualidade. Valendo-se do trabalho de Anderson e Tushman (1986) ${ }^{3}$, ele afirma (e demonstra empiricamente) que o novo modelo de qualidade representa uma descontinuidade tecnológica no que se fazia, e que implicou a destruição de competências e rompimento dos padrões existentes dominantes nas estruturas da indústria. O novo modelo exigiu forte mudança nas habilidades, competências e organização dos processos de produção. E levou muitos anos para que a indústria ocidental entendesse o que qualidade agora compreendia.

\section{Os MEGs amparados pelos Prêmios Nacionais da Qualidade}

Segundo Pagliuso e Cardoso (2005), nos conceitos do TQM se fortaleceram os esforços no desenvolvimento de orientações ao gerenciamento das empresas, por meio de requisitos sobre como as organizações deveriam ser conduzidas de uma forma sistêmica. Identificar e avaliar a eficácia das organizações foi uma conseqüência desses esforços. Com base em Harrison e Shiron (1999) ${ }^{4}$, estes autores discorrem que, para que seja possível verificar a eficácia de uma organização é necessário definir um modelo de referência para viabilizar a comparação da situação desejada com as informações coletadas na organização. 
A introdução da concepção de excelência gerencial nos institutos e centros de pesquisa brasileiros - o projeto excelência na pesquisa tecnológica

Para Miguel (2005), a evolução no conceito do TQM veio com a necessidade de incorporar os diversos interesses das partes envolvidas (stakeholders) de uma organização na busca da excelência em desempenho. Em um passado recente, o acionista era a principal parte interessada em seu desempenho, sendo-lhe dispensada grande parcela de atenção e importância. Com a alteração desse enfoque, ganham destaque outros indivíduos/grupos afetando os resultados de uma organização (ou afetados por), tais como a força de trabalho, os fornecedores, os clientes e os parceiros, cujos papéis deveriam ser incluídos nos modelos de excelência (KANJI, 2002).

Com o aumento na importância da qualidade na indústria e em outros setores, como governo e educação, tem havido uma ênfase sobre programas de premiação da qualidade, especialmente a partir de meados da década de 80. Tais prêmios representam um reconhecimento a organizações que alcançam objetivos de melhoria da qualidade (CARTIN, 1999).

\subsection{Evolução dos modelos de excelência: o Prêmio Deming, o MBNQA e o EQA}

O precursor desses prêmios foi o prêmio japonês (Deming Prize), instituído no Japão, na década de 1950. Em 1987, os EUA desenvolveram o Malcolm Baldrige National Quality Award (MBNQA). Em 1991, foi estabelecido o European Quality Award (EQA). Hoje há mais de 75 prêmios relativos à qualidade, todos eles referentes à busca de melhores níveis de qualidade e produtividade, com muitos temas e elementos em comum. Conforme Tan (2002), o movimento de prêmios nacionais de qualidade pelo mundo inteiro é, ainda, relativamente jovem, com grande parte deles tendo menos de uma década de existência e muitos outros se encontrando em distintos estágios de desenvolvimento.

O interesse ampliado de organizações no mundo inteiro por processos de avaliação e diagnóstico de práticas e resultados organizacionais se deve à introdução dos modelos de excelência americano e europeu, cujos critérios condensam um abrangente e holístico modelo de gestão cobrindo várias atividades, práticas e processos organizacionais, bem como fornecem um mecanismo para diagnosticar o estado atual de uma organização quanto ao desenvolvimento da TQM (DALE, 2003).

Assim, os modelos de excelência compreendidos em um prêmio de qualidade em nível nacional visam avaliar uma organização em relação às práticas gerenciais por ela utilizadas, 
Marconi Edson Esmeraldo Albuquerque \& Maria Beatriz Machado Bonacelli

bem como seus resultados organizacionais. Além desse, tais modelos têm como objetivos: auxiliar o desenvolvimento de práticas de melhoria do desempenho das organizações; disseminar boas práticas para outras organizações; e servir como ferramenta para transmitir conhecimentos em desempenho gerencial, planejamento, treinamento e avaliação.

A adoção de modelos de excelência, na visão de quem advoga sua aplicação, pode assim, trazer várias possibilidades às organizações aplicantes (sejam elas pequenas ou grandes, com ou sem fins lucrativos, públicas ou privadas, do ramo industrial ou de serviços, hospitais, escolas, ...) como a ampliação de seus níveis de eficiência operacional, constituição de referenciais para outras organizações, bem como especialização de seu corpo funcional. Em essência, tais modelos representam uma ferramenta para medição e avaliação do desempenho das organizações, ajudando-as a focar seus esforços de melhoria sobre áreas pré-estabelecidas sendo, em grande medida, baseado em teorias de TQM (KANJI, 2002).

\section{O Prêmio da Qualidade Japonês (Deming Prize)}

O Deming Prize $e^{5}$ foi instituído em 1951, pela Japanese Union of Scientists and Engineers (JUSE), em honra de W.E. Deming, por suas contribuições ao desenvolvimento da causa da qualidade industrial no Japão. O Prêmio foi desenvolvido para garantir que bons resultados fossem alcançados por meio da implementação de atividades de Controle de Qualidade por Toda a Empresa (CWQC - Company-wide Quality Control), e está baseado na aplicação de um conjunto de princípios e técnicas estatísticas (DALE, 2003). Esse prêmio é significante por duas razões: foi o primeiro a introduzir a prática da auto-avaliação e os conceitos de pontuação e visitas aos aplicantes em um processo de premiação, que é a forma como o MBNQA e o EQA operam (PORTER; TANNER, 2004).

Embora Deming tenha emprestado seu nome ao prêmio japonês, ele jamais teve participação ativa na criação ou gestão do mesmo. Sua principal ligação com o prêmio era o patrocínio nos primeiros anos, oriundo da doação de recursos advindos da venda de materiais de sua autoria e dos direitos de tradução de seus livros. Deming, pelo contrário, não era favorável à ideia de prêmios de qualidade. Como relatam Porter e Tanner (2004), ele sentia que prêmios de qualidade criam vencedores e perdedores, e que a conquista de um prêmio pode ser vista como o final da jornada. Ele via a melhoria contínua como um processo incessante.

O Deming Prize está baseado nos seguintes critérios: políticas, organização, informação, 
A introdução da concepção de excelência gerencial nos institutos e centros de pesquisa brasileiros - o projeto excelência na pesquisa tecnológica

padronização, recursos humanos, garantia de qualidade, manutenção, melhoria, efeitos (resultados) e planos futuros.

\section{O Prêmio Americano da Qualidade (MBNQA)}

A criação do prêmio americano se insere em um contexto social e institucional amplo em que os EUA, assombrados com a perda de competitividade de sua indústria frente aos japoneses, nos anos 80, engendraram uma densa rede institucional de aprendizado e difusão do ideário da qualidade. Essa rede de acumulação de expertise não se voltou apenas para a adoção de uma abordagem específica para a melhoria da qualidade, mas se constituiu em uma vasta infraestrutura de organizações e atividades para identificação e difusão de novas práticas de qualidade. Dela faziam parte usuários (organizações), consultores e vários atores de fora do mercado, tais como associações profissionais, órgãos de governo, iniciativas público-privadas, universidades e organizações de pesquisa independentes. Na visão de Cole (1999), esses e muitos outros atores vieram a se constituir uma comunidade de inovação e difusão, que ajudou muitos gerentes na busca por práticas efetivas, várias delas oriundas de abordagens japonesas e adaptadas ao contexto americano ${ }^{6}$.

O MBNQA foi instituído em 1987, pela Lei Pública Malcolm Baldrige National Quality Improvement Act of 1987, e era uma daquelas atividades institucionais de âmbito nacional que compunha a infraestrutura americana em prol da qualidade. Para Cole (1999), ele foi uma grande inovação institucional e a mais importante inclusão à infraestrutura nacional para identificar e difundir boas práticas em qualidade. Esse autor ressalta, ainda, que o MBNQA deu uma grande contribuição à criação de um 'projeto dominante' sobre como fazer melhoria da qualidade e se tornou, por vários anos, a força direcionadora de um movimento nacional.

O Programa Baldrige resultou de esforços cooperativos entre governo e iniciativa privada e tinha como proposta promover a conscientização de que excelência em desempenho é um elemento importante para a competitividade e promover o compartilhamento de informação sobre estratégias de desempenho bem sucedidas e os benefícios advindos da sua adoção (DALE, 2003; PORTER; TANNER, 2004).

O Baldrige foi inspirado pelo Deming Prize e ambos guardam semelhanças em seus princípios e critérios de avaliação. Conforme Porter e Tanner (2004), o modelo de excelência americano é o mais conhecido no mundo e o mais utilizado em processos de auto-avaliação, 
Marconi Edson Esmeraldo Albuquerque \& Maria Beatriz Machado Bonacelli tendo influenciado muitas premiações internacionais de excelência em desempenho.

O Prêmio consiste em um sistema de pontuação ponderada de sete categorias de critérios de desempenho - 1) liderança; 2) planejamento estratégico; 3) foco no cliente e no mercado; 4) medição, análise e gestão do conhecimento; 5) foco nos recursos humanos; 6) gestão dos processos; 7) resultados da organização - que estão baseados em um conjunto de valores e conceitos que estão incorporados em organizações bem sucedidas. Cada critério tem uma pontuação pré-estabelecida e, juntos, totalizam 1000 pontos. Os sete critérios estão subdivididos em itens que consistem de uma ou mais áreas que deveriam ser avaliadas na organização.

A pontuação é atribuída aos itens e baseia-se em três dimensões de avaliação: 1) abordagens; 2) implementação; e 3) resultados. As abordagens referem-se aos métodos que a organização aplicante usa para atender aos requisitos de cada item. A implementação diz respeito à extensão em que as abordagens são aplicadas a todas as áreas e atividades relevantes. Os resultados são conseqüência do atendimento dos requisitos de cada item (PORTER; TANNER, 2004). Dessa forma, uma organização que adota tal sistema de avaliação deveria abordar todos os itens, implementá-los com acompanhamento e analisar seus resultados. O modelo empregado no Baldrige é, também, uma ferramenta aplicada para auto-avaliação e é com esse objetivo que muitas organizações usam os critérios de excelência, sem qualquer intenção de se candidatar a um prêmio.

\section{O Prêmio Europeu da Qualidade (EQA)}

O European Quality Award (EQA) foi criado em 1991 e é um equivalente europeu do MBNQA. Em sua concepção, seus idealizadores receberam influência tanto da experiência do MBNQA como do Prêmio Deming, tendo sido refinado desde então (PORTER; TANNER, 2004). Sua origem apresenta contexto semelhante ao da criação do prêmio americano, qual seja, a preocução de muitos países ocidentais, no início dos anos 1980, com os baixos níveis de produtividade industrial demonstrados na maior parte das economias nacionais, quando comparadas com os japoneses. Influenciados por esse contexto e vislumbrados com a rápida difusão dos conceitos propostos no MBNQA nas empresas americanas, 14 executivos de empresas líderes européias formaram a European Foundation for Quality Management (EFQM) com o prósito de melhorar a posição competitiva das empresas européias no mercado mundial (PORTER; TANNER, 2004). A EFQM consiste no centro de uma rede em prol do 
A introdução da concepção de excelência gerencial nos institutos e centros de pesquisa brasileiros - o projeto excelência na pesquisa tecnológica

ideário da excelência em desempenho e é apoiada por muitas organizações congêneres em toda a Europa. A maioria dos países europeus adota o modelo de excelência e o processo de premiação da EFQM para seus prêmios nacionais.

Criado por gerentes de empresas-líderes, acadêmicos e consultores, sob os auspícios da EFQM e da European Organization for Quality (EOQ) e apoiado pela Comissão Européia, o prêmio europeu foi planejado para focalizar atenção sobre excelência organizacional, fornecer estímulo para companhias desenvolverem iniciativas de melhoria organizacional e demonstrar resultados alcançáveis em todos os aspectos de atividade organizacional (DALE, 2003).

$\mathrm{O}$ modelo de excelência que orienta aquele prêmio está baseado em oito conceitos fundamentais: orientação para resultados; foco no cliente; liderança e constância de propósitos; gestão por processos e fatos; desenvolvimento e envolvimento das pessoas; aprendizagem contínua; inovação e melhoria; desenvolvimento de parcerias; e responsabilidade social corporativa. Tais conceitos são desdobrados em nove critérios que as organizações podem usar para avaliar e medir seu desempenho: liderança; política e estratégia; pessoas; parceiros e resursos; processos; resultados clientes; resultados pessoas; resultados sociedade; resultados chave do desempenho.

\subsection{O modelo brasileiro de excelência gerencial do Prêmio Nacional da Qualidade}

Diversos países criaram seus programas de estímulo à excelência organizacional tomando por base os modelos americano, europeu e o Deming Prize, definindo, assim, prêmios nacionais e modelos de referência em gestão (TAN, 2002; TAN et al, 2003).

No Brasil, um grupo formado por profissionais de distintos setores (indústria, serviços, consultoria e academia) iniciou um estudo em 1989 sobre o desenvolvimento de um prêmio brasileiro nos moldes daqueles existentes mundo afora (MIGUEL, 2005). Diversos modelos de premiações no Brasil e no mundo foram estudados, com destaque para o Deming Prize e para o MBNQA. Foi criada, então, a Fundação para o Prêmio Nacional da Qualidade (FPNQ), hoje Fundação Nacional da Qualidade (FNQ), que é uma entidade privada, sem fins lucrativos, instituída por 39 organizações nacionais e transnacionais para administrar o PNQ. Este, por sua vez, teve seu primeiro ciclo em 1992.

Conforme Souza et. al (2005), houve apoio financeiro e institucional do Ministério da Ciência e Tecnologia (MCT) para a criação da FNQ e, segundo pesquisa de campo 
Marconi Edson Esmeraldo Albuquerque \& Maria Beatriz Machado Bonacelli empreendida por Darós (1999), o Prêmio Nacional consistia em um dos projetos do Programa Brasileiro da Qualidade e Produtividade (PBQP) ${ }^{7}$.

$\mathrm{O}$ MEG da FNQ é um instrumento em apoio à avaliação, diagnóstico e desenvolvimento dos sistemas de gestão das organizações, e pode ser usado tanto para auto-avaliação e obtenção de um diagnóstico organizacional, como para candidatura de organizações ao PNQ.

O MEG/FNQ constitui um modelo de gestão adotado por muitas organizações brasileiras e baseia-se em 11 conceitos fundamentais relacionados à excelência em gestão (FNQ, 2008), sobre os quais as organizações devem estruturar seus sistemas de gestão. São eles: pensamento sistêmico; aprendizado organizacional; cultura de inovação; liderança e constância de propósitos; orientação por processos e informações; visão de futuro; geração de valor; valorização das pessoas; conhecimento sobre o cliente e o mercado; desenvolvimento de parcerias; responsabilidade social. Estes conceitos são desdobrados em 8 critérios de excelência: 1) liderança; 2) estratégias e planos; 3) clientes; 4) sociedade; 5) informação e conhecimento; 6) pessoas; 7) processos; 8) resultados. Juntos, esses critérios compõem a estrutura do MEG/FNQ (Figura 1).

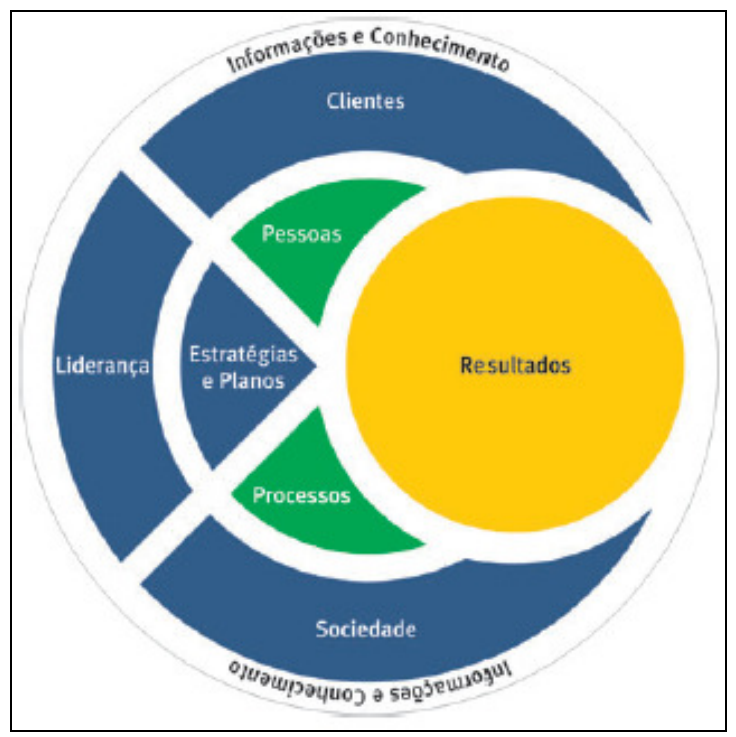

Figura 1 - Modelo de Excelência da Gestão da FNQ

Fonte: FNQ (2008)

Cada critério é composto por itens que contêm os requisitos para avaliação. Na edição 2009 dos Critérios de Excelência do PNQ (CE-PNQ), os oito critérios estão desdobrados em 24 itens, cada um possuindo requisitos específicos e uma pontuação máxima. Destes, 18 são itens de processos gerenciais e 6 de resultados organizacionais. Os itens de processos gerenciais solicitam das organizações aplicantes informações relacionadas ao sistema de 
A introdução da concepção de excelência gerencial nos institutos e centros de pesquisa brasileiros - o projeto excelência na pesquisa tecnológica

gestão da organização, sem prescrever práticas de gestão, métodos de trabalho ou ferramentas. Os itens de resultados organizacionais solicitam a apresentação dos resultados, informações comparativas e explicações sobre eventuais tendências ou níveis adversos (FNQ, 2008). No geral, o PNQ totaliza 1000 pontos, distribuídos irregularmente entre os critérios.

\section{Difusão de Modelos de Excelência Gerencial nos ICPs brasileiros}

Tal qual o caso americano, no Brasil tem havido grande disseminação dos CE-PNQ em diversos setores da sociedade, além da iniciativa privada (e.g. Serviço Público, Polícia Militar, Exército, Saneamento, Correios). A FNQ vem estimulando a criação de premiações estaduais, regionais e setoriais e tem participado da articulação da Rede Nacional da Gestão Rumo à Excelência, que engloba 54 programas e prêmios regionais e setoriais que utilizam o MEG/FNQ como norteador de seus processos de avaliação (FNQ, 2009). Um desses programas de estímulo ao desenvolvimento de sistemas de gestão organizacional, que assumiu como modelo de referência os CE-PNQ, é o Projeto Excelência na Pesquisa Tecnológica, que será tratado nesta seção.

\subsection{Modernização da gestão de ICPs atendendo às transformações no Sistema de C,T\&I}

A contribuição da inovação para o crescimento econômico é uma questão fundamental para a competitividade das economias nacionais. Muitos governos têm buscado expandir suas capacidades científicas e de inovação (como forma de promover a atração de investimentos externos em P\&D, aliado à criação de um ambiente fiscal favorável) e têm aumentado o apoio à pesquisa no setor público, embora haja restrições em alguns países. O gasto público com P\&D (relativo ao PIB) aumentou modestamente nos países desenvolvidos, com a intensidade da $P \& D$ universitária crescendo mais rapidamente do que aquela realizada em outras organizações de pesquisa. Países em desenvolvimento, em seu conjunto, têm ampliado sua participação na P\&D mundial, especialmente a China ${ }^{8}$ (OECD, 2008), que tem demonstrado compromisso político em estimular o financiamento em P\&D e inovação.

As políticas de C,T\&I estão evoluindo em resposta a reformas mais amplas para incrementar a produtividade e o crescimento econômico (políticas de competitividade), atendendo a preocupações nacionais (e.g. empregos, saúde e educação) e aos desafios globais (como segurança energética e mudanças climáticas), que constituem desafios aos países. $\mathrm{O}$ 
Marconi Edson Esmeraldo Albuquerque \& Maria Beatriz Machado Bonacelli aumento da globalização da $\mathrm{P} \& \mathrm{D}$ e a rápida difusão de formas mais abertas e compartilhadas de inovação, também representam importantes desafios à constituição de políticas nesta área. A crescente complexidade relativa às atividades de $\mathrm{C}, \mathrm{T} \& \mathrm{I}$, bem como a previsível convergência entre essas políticas, exigem um maior esforço de coordenação e impõem a necessidade de revisão das estruturas de governança. Tais mudanças são refletidas nas reformas das instituições e mecanismos de governança em áreas como C,T\&I (OECD, 2008).

ICPs desempenham importante papel nos sistemas de inovação, sendo fundamentais na criação e difusão de conhecimento tecnológico. Em muitos países, essas instituições vêm passando por transformações, redefinindo papéis e funções, buscando novas formas de gestão, tudo isso reflexo de mudanças na dinâmica global da P\&D e da inovação.

Outra tendência verificada no documento de perspectivas em C, T\&I da OCDE (OECD, 2008), e que seguramente traz implicações para a atuação de ICPs, é a crescente importância de as políticas de inovação: i) ampliarem seu escopo além da construção de capacidades (supply-side) - que focalizam a comercialização ou transferência de resultados da pesquisa pública para a indústria - dando atenção, também, para a procura de inovações (demand-side) - que se baseia no desenvolvimento conjunto, frequentemente via parcerias público-privadas, envolvendo redes de organizações; e ii) irem além do apoio à inovação tecnológica, considerando-se, também, outros tipos de inovação (organizacionais e de marketing), setores pouco intensivos em P\&D (bens tradicionais e aqueles baseados em recursos) e o setor de serviços. Dadas as mudanças globais em relação a P\&D e inovação, focalizar as políticas sobre o lado da oferta não é mais suficiente. Desenvolver políticas que apoiem tanto a oferta quanto a demanda por inovação passa a ser a tônica nos países desenvolvidos.

No caso do Brasil, o desafio estrutural é aumentar a contribuição da inovação para o crescimento da produtividade e competitividade e o principal desafio da política de inovação é encorajar as empresas a inovar. A política de inovação no Brasil vem incorporando uma visão mais abrangente, de modo a se aproveitar de sinergias potenciais entre promoção de C\&T, suporte à P\&D e estímulo à competitividade comercial (OECD, 2008).

Os ICPs estão sendo compelidos, pois, a adotar novas estratégias de ação, buscando explorar variadas frentes de atuação. Tal situação impõe, dentre outras coisas, decisões relativas às relações que mantêm interna e externamente à sua estrutura - com o Estado, com a sociedade e com outros atores do sistema de inovação (BONACELLI, 2004). Por conseguinte, é premente a necessidade de ICPs reestruturarem seus sistemas de gestão e construírem capacidades para melhor desempenharem seus papéis e funções em face das 
A introdução da concepção de excelência gerencial nos institutos e centros de pesquisa brasileiros - o projeto excelência na pesquisa tecnológica

transformações nas políticas de promção da C,T\&I e competitividade.

Nesse intento, seguindo tendência internacional de profissionalização da gestão desse tipo de organização, vários ICPs no país têm adotado modelos de excelência gerencial, visando a modelagem de seus sistemas gerenciais.

\subsection{A adoção de MEGs nos ICPs brasileiros}

A Associação Brasileira das Instituições de Pesquisa Tecnológica (ABIPTI) é uma entidade de direito privado, sem fins lucrativos, e foi fundada no início da década de 1980 com o objetivo de intensificar a participação dos institutos de pesquisa tecnológica industrial na política de desenvolvimento nacional. Como resposta às dificuldades percebidas pelos ICPs na segunda metade dos anos 1990, a ABIPTI propôs o Projeto Excelência na Pesquisa Tecnológica (PEPT), que teve início em maio de 1998 (e continua existindo até hoje), e se pautava essencialmente em viabilizar a internalização do MEG/FNQ nos ICPs, bem como a criação de um banco de indicadores de desempenho dessas organizações, visando com isso promover a melhoria do desempenho dos institutos adotantes e estimular a criação e/ou aprimoramento de suas práticas de gestão. Tal estratégia foi implementada pela ABIPTI, com apoio de um grupo de institutos ${ }^{9}$, do suporte técnico (remunerado) de consultores da FNQ e o concurso de recursos financeiros liberados pelo CNPq.

Além da busca da melhoria do desempenho gerencial dos ICPs, por meio do estímulo à adoção dos CE-PNQ e uso de indicadores (comparativos) de desempenho dessas organizações, o PEPT tinha como metas: i) viabilizar a internalização de práticas de gestão da qualidade nos ICPs; ii) gerar informações sobre o desempenho dos ICPs como subsídio ao planejamento e a formulação de políticas para o setor; e iii) promover a implementação de planos de melhoria nos ICPs.

O Gráfico 1 apresenta a evolução dos números de adesões e de institutos avaliados do PEPT. 
Marconi Edson Esmeraldo Albuquerque \& Maria Beatriz Machado Bonacelli

Gráfico 1 - Evolução do Projeto Excelência na Pesquisa Tecnológica: Número de ICPs Adesos e Avaliados, 1999 - 2007

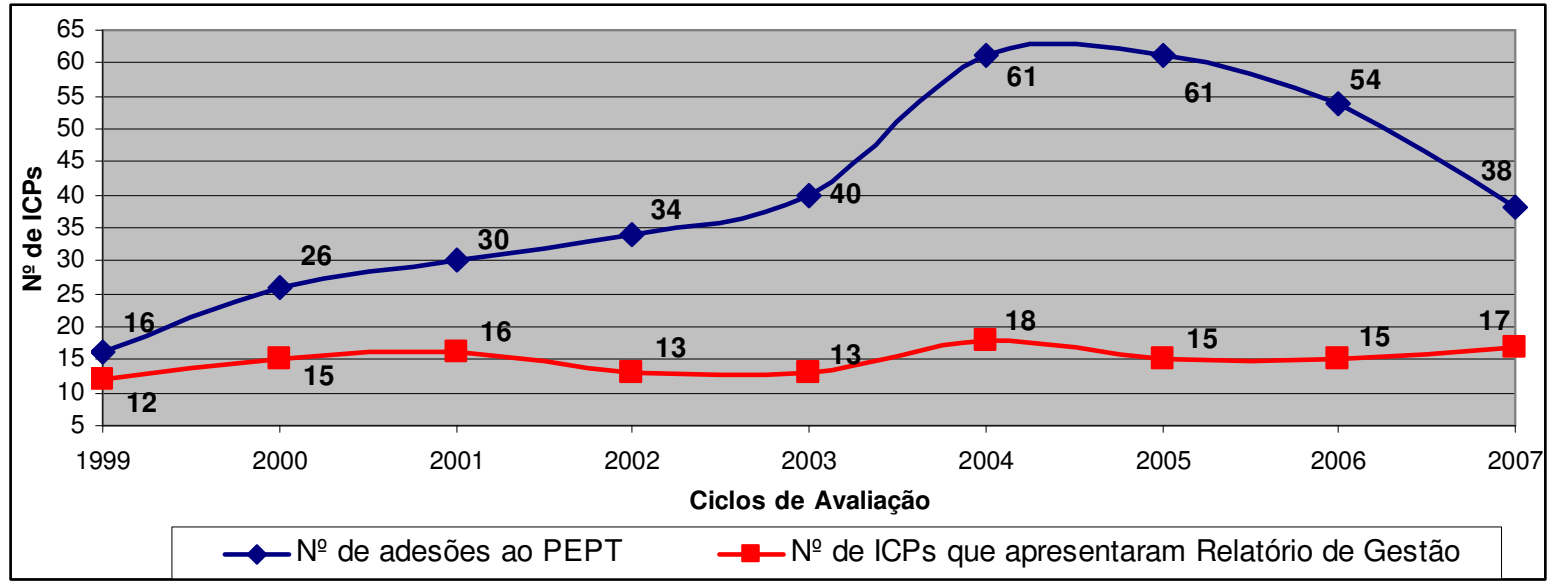

Fonte: Elaboração própria, a partir de dados obtidos no endereço: http://www.sgid.abipti.org.br/Indicadores.htm.

No período de 1999 a 2005, houve progressiva adesão de ICPs ao PEPT (alguns deles aderiram à $\mathrm{ABIPTI}$ devido àquele projeto $^{10}$ ), inclusive institutos mais voltados à pesquisa básica. Ao longo de nove ciclos de avaliação (período no qual 101 ICPs participaram de algum modo das atividades do PEPT) houve uma participação média de 40 instituições por ciclo. Em 2007, último ciclo de avaliação ocorrido ${ }^{11}$, houve uma adesão de 38 institutos de pesquisa assim distribuídos: unidades de pesquisa da Embrapa (10); ligados ao Ministério da Ciência e Tecnologia (MCT) (08); estaduais (05); federais (06); cativos de empresas (02); privados (07). No entanto, nos últimos anos tem havido perda de interesse pelo PEPT, percebida a partir da queda no número de adesões nos últimos dois ciclos. Além disso, a evolução no número de institutos avaliados - com tendência à constância - tem evidenciado pouca atratividade dos institutos participantes pelo processo de avaliação proposto no PEPT. Em média, 15 institutos foram avaliados por ano no período 1999 - 2007, bem abaixo da média anual de 40 institutos adesos no mesmo período.

Isto fica melhor evidenciado no Gráfico 2, que mostra, em termos percentuais, a razão entre o número de institutos avaliados e o número de institutos que aderiram ao PEPT. 
A introdução da concepção de excelência gerencial nos institutos e centros de pesquisa brasileiros - o projeto excelência na pesquisa tecnológica

Gráfico 2 - Projeto Excelência na Pesquisa Tecnológica: Relação Percentual de Institutos Avaliados / Institutos Adesos - 1999 / 2007

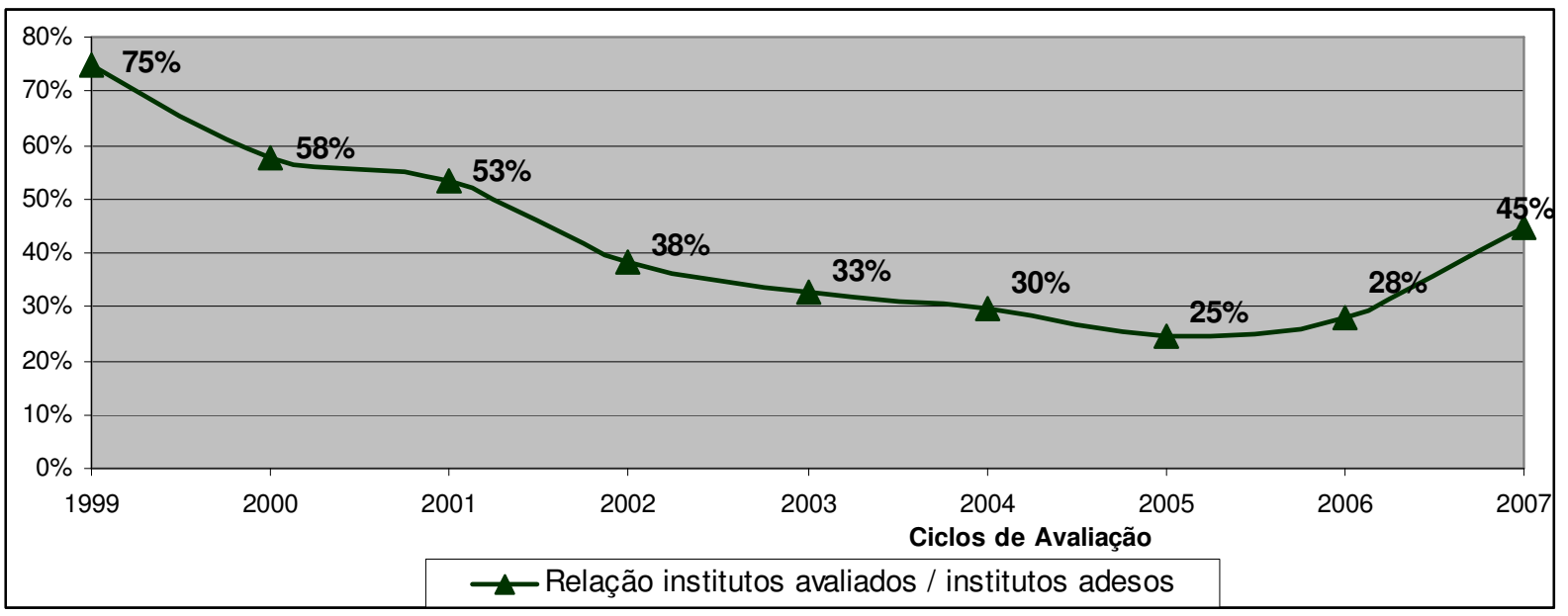

Fonte: Elaboração própria, a partir de dados obtidos no endereço: http://www.sgid.abipti.org.br/Indicadores.htm.

Percebe-se variação decrescente no período 1999 - 2005, reflexo do número praticamente constante de institutos avaliados, bem como da acentuada procura pelo PEPT nesse período. No período 2006 - 2007, a curva mostra aparente melhora na relação avaliação/adesão. Todavia, ela é ilusória, considerando-se que no período houve queda nas adesões ao projeto da ABIPTI. A evolução desses dados sugere, também, que houve uma maior concentração de esforços na busca de novas adesões, em detrimento à internalização do MEG/PNQ nos institutos, que refletiria num número maior de relatórios submetidos à avaliação do PEPT.

\subsection{Contexto de criação do PEPT}

Conforme mencionado, um conjunto de transformações tem impactado os ICPs nas últimas décadas. Para se discutir o contexto de criação do PEPT, faz-se necessário entender como a ABIPTI percebia esse ambiente de mudanças, bem como os impactos sobre os ICPs. Segundo consta na primeira versão do documento do PEPT (mar./1997), proposto ao CNPq (ABIPTI, 1998), a desregulamentação e liberalização da economia nacional à competição externa e seus conseqüentes efeitos sobre a indústria local haviam provocado a necessidade de adaptações e mudanças institucionais em muitos dos instrumentos de promoção e fomento tecnológico, especialmente institutos de pesquisa. Os seguintes aspectos foram tidos como impactantes às atividades dessas organizações: i) rápidas mudanças técnicas; ii) crescente 
Marconi Edson Esmeraldo Albuquerque \& Maria Beatriz Machado Bonacelli importância do comércio internacional; iii) redução das barreiras a importações; iv) sistemas produtivos e comerciais globalizados; v) redução do papel do Estado; vi) redução dos recursos governamentais para a pesquisa tecnológica; vii) crescente importância atribuída ao capital estrangeiro; ix) preocuções com o meio ambiente.

A indústria nacional, que até o início dos anos 1990 não havia experimentado concorrência externa expressiva, viu-se obrigada a adaptar-se rapidamente às novas condições que nesse contexto se apresentavam, sob pena de não serem suficientemente competitivas perante as empresas estrangeiras. Ela precisava de auxílio para compreender as novas regras vigentes na economia internacional e transformar suas estruturas e sistemas visando se adequar. Outro aspecto que depunha contra a empresa nacional era sua vulnerabilidade em produzir/incorporar conhecimento tecnológico em seus sistemas produtivos, sendo este um dos principais fatores que alimentava a competição mundial. Visando suprir essas imperfeições, havia a necessidade de fortalecer e reestruturar os institutos, "para que os mesmos pudessem fornecer insumos e subsídios para promover o desenvolvimento da indústria nacional no novo contexto" (MOTOYAMA; MARQUES; QUEMEL, 2008, p.49).

No documento do PEPT é recorrente a atribuição dos problemas dos institutos no contexto dos anos 1990 a deficiências na sua relação com o setor produtivo, além da diminuição de recursos de seus mantenedores. Conforme aquela fonte, não existia a cultura de os institutos irem à procura de clientes, identificar e atender suas necessidades. Predominava uma atitude passiva, em que se esperava que algum cliente viesse consultar sobre a especialidade de cada instituto. Além disso, embora disposto em muitos estatutos, geralmente não foram estruturadas nos institutos áreas de marketing, comercialização ou vendas (ABIPTI, 1998). Porém, não se pode atribuir toda essa carga apenas aos institutos. Conforme Bell e Pavitt (1995), os encadeamentos entre institutos de P\&D e as atividades de produção das empresas dependem pesadamente do vigor das capacidades tecnológicas dentro das próprias empresas. Para a empresa nacional, que acabara de sair de um modelo baseado na proteção à competição estrangeira, dispor dessa capacidade de absorção parecia ser algo pouco praticado.

Uma das ações empreendidas pela ABIPTI foi o Programa de Revitalização dos Institutos de Pesquisa Tecnológica (REVITE), cujo pressuposto básico era a necessidade de reorientar os institutos industriais para atender as demandas do setor produtivo de prestação de serviços técnicos especializados e de atividades de P\&D. Segundo Motoyama, Marques e Quemel (2008), esse programa focava a ideia de reengenharia para alcançar a qualidade. Seu escopo 
A introdução da concepção de excelência gerencial nos institutos e centros de pesquisa brasileiros - o projeto excelência na pesquisa tecnológica

era a reformulação das estruturas administrativas e operacionais dos institutos, capacitando-os para o cumprimento de seu novo papel, estratégico na modernização do parque industrial brasileiro e na melhoria da qualidade, produtividade e competitividade de empresas nacionais.

Em 1995, ao entrar em curso uma nova gestão na ABIPTI, esta abordou, dentre outos, o seguinte ponto: desenvolver indicadores para medir desempenho nos institutos (MOTOYAMA; MARQUES; QUEMEL, 2008), indicando, assim, o rumo que tomaria a Associação no sentido de promoção do desenvolvimento gerencial das instituições a ela associadas.

Todavia, apenas em 1997 a questão da análise do modelo de gestão dos institutos de pesquisa entrou de modo destacado na agenda da ABIPTI. Em abril daquele ano, foi realizado o seminário "Excelência na pesquisa tecnológica", em parceria com a FNQ, o Instituto Brasileiro de Informação em Ciência e Tecnologia (IBICT) e o Conselho Britânico. Participaram do evento 30 dirigentes de entidades associadas à ABIPTI, bem como representantes de outros atores do SNI, tais como CNPq, FINEP, MCT e SEBRAE. Como apresentado no início desta seção, no ano seguinte, no âmbito do REVITE, iniciou-se o primeiro ciclo de atividades do PEPT. Vislumbrava-se, assim, o MEG/FNQ, admitindo-se a possibilidade de adaptações, como uma promessa para o desenvolvimento dos $\operatorname{ICPs}^{12}$.

\section{Considerações finais}

A qualidade e sua gestão têm se transformado desde o início do século passado, assumindo novas funções e atividades, resultantes do amadurecimento na compreensão do que é qualidade, bem como das transformações no mundo da produção. Dentre os elementos mais recentes do processo evolutivo da qualidade, emergindo a partir da pressão competitiva decorrente das técnicas 'orientais' de produção, surge o Malcolm Baldrige National Quality Award, como um meio de identificar e difundir boas práticas em qualidade. Esse modelo vem sendo reproduzido mundo afora, propagando as ideias e diretrizes presentes na TQM, para além do setor industrial, alcançando áreas como Governo, Serviços, Saúde e Educação, entre outras.

A experiência da ABIPTI na adoção do MEG/PNQ como forma de modernizar a gestão dos institutos de pesquisa tem demonstrado dificuldade em difundir a ideia de 'excelência' em gestão entre esses atores do Sistema de Inovação, seja por conta da forma como esse projeto 
Marconi Edson Esmeraldo Albuquerque \& Maria Beatriz Machado Bonacelli

foi concebido, desenvolvido e implementado, seja pela dificuldade encontrada pelos próprios ICPs em internalizar em suas estruturas novos processos gerenciais e institucionais (SALLESFILHO; BONACELLI, 2007) - e isso por vários e diferentes motivos.

Um maior envolvimento de ICPs em modelos de modernização em gestão seria mais efetivo se as iniciativas levassem em conta as diferenças - de várias ordens - entre essas instituições, dentre elas, o status jurídico (a quem a organização está institucionalmente vinculada - no caso o setor público, administração direta, indireta, empresa pública, organização social, entre outras); proximidade com instâncias governamentais (se elaboram e/ou executam funções públicas, por exemplo); o tipo de atividades de C\&T que desenvolvem - mais científicas, mais tecnológicas, de prestação de serviços, tecnologias industriais básicas (TIB), atividades de C\&T correlatas; com que áreas do conhecimento mais se relacionam; existência e tipo de vínculos e parcerias externas; em que regiões do país atuam; a incidência de leis de incentivos em suas atividades; o nível de concorrência que sofrem de outros tipos de instituições (públicas, privadas, nacionais, internacionais etc.), entre outras variáveis.

Entretanto, esses atributos não dizem tudo, pois instituições com perfis semelhantes - as organizações estaduais de pesquisa agropecuária, por exemplo - podem apresentar (e têm apresentado) desempenho muito desigual frente a situações similares de risco e de oportunidades. Isso porque algumas delas passaram a profissionalizar suas atividades de gestão - em várias frentes - justamente para enfrentar (e algumas, se antecipar) às mudanças no ambiente de se fazer e desenvolver pesquisa. Em alguns casos, o PEPT teve influência nessas transformações, mas acredita-se que o alcance dessa iniciativa poderia ter sido maior, caso o diagnóstico das transformações do ambiente da P\&D tivesse sido mais preciso e a implementação e aprimoramento dos processos de gestão tivessem sido mais bem instrumentalizados.

\section{REFERÊNCIAS}

ABIPTI. Projeto excelência na pesquisa tecnológica: implantação do modelo de gestão do prêmio nacional da qualidade nos institutos de pesquisa tecnológica. In: CONSELHO NACIONAL DE DESENVOLVIMENTO CIENTÍFICO E TECNOLÓGICO - CNPq. Processo No 680.048/1998-7. Brasília: CNPq, maio 1998.

BELL, M., PAVITT, K. The development of technological capabilities. In: IRFAN-ULHAQUE. Trade, technology, and international competitiveness. Washington: World Bank, 
A introdução da concepção de excelência gerencial nos institutos e centros de pesquisa brasileiros - o projeto excelência na pesquisa tecnológica

1995. cap. 4, p. 69-101.

BONACELLI, M. B. M. Institutos e centros de p\&d: situação atual e avaliação do potencial inovativo. Campinas: Unicamp/DPCT/GEOPI. Programa Nacional de Apoio à Administração Fiscal para os Estados Brasileiros. Projeto BRA/97/032 - IPEA - PNUD. Relatório Final. 2004.

CARTIN, T.J. Principles and practives of organizational performance excellence. Milwaukee: ASQ, 1999.

COLE, R.E. Managing quality fads: how american business learned to play the quality game. New York: Oxford University Press, 1999.

DALE, B.G. Managing quality. 4.ed. Malden - Oxford - Melbourne - Berlin: Blackwell Publishing, 2003.

DARÓS, M.da M. O programa brasileiro da qualidade e produtividade: uma análise de política. 1997. 167f. Dissertação (Mestrado em Política Científica e Tecnológica) - Instituto de Geociências, Universidade Estadual de Campinas, Campinas.

FNQ. Critérios compromisso com a excelência e rumo à excelência 2009-2010. São Paulo: Fundação Nacional da Qualidade, 2009.

. Critérios de Excelência 2009; avaliação e diagnóstico da gestão organizacional. São Paulo: Fundação Nacional da Qualidade, 2008.

GARVIN, D.A. Gerenciando a qualidade; a visão estratégica e competitiva. Rio de Janeiro: Qualitymark, 1992.

KANJI, G.K. Measuring business excellence. London - New York: Routledge, 2002.

MIGUEL, P.A.C. Gestão da qualidade; TQM e modelos de excelência. In: CARVALHO, Marly M., PALADINI, Edson P. (Orgs.) Gestão da qualidade; teoria e casos. Rio de Janeiro: Campus, 2005. pp. 85-124.

MOTOYAMA, S., MARQUES, P.Q., QUEMEL, M.A.R.. Uma associação para a tecnologia brasileira; Abipti 25 anos. 2.ed. Brasília: ABIPTI, 2008. 
Marconi Edson Esmeraldo Albuquerque \& Maria Beatriz Machado Bonacelli

OECD. Science, Technology and Industry Outlook 2008. Paris: OECD, 2008.

PAGLIUSO, A.T.; CARDOSO, R. Gestão organizacinal: passado, presente e futuro. In: Ministério da Ciência e Tecnologia, Confederação Nacional da Indústria, Serviço Nacional de Aprendizagem Industrial, Instituto Euvaldo Lodi. Tecnologia industrial básica; trajetória, tendências e desafios no Brasil. Brasília: MCT, CNI, SENAI/DN, IEL/NC, 2005. pp. 149-161.

PORTER, L.J., TANNER, S.J. Assessing business excellence: a guide to business excellence and self-assessment. 2.ed. Oxford - Burlington: Elsevier Butterworth-Heinemann, 2004.

SALLES-FILHO, S.; BONACELLI, M. B. Em busca de um novo modelo para as organizações públicas de pesquisa no Brasil. Ciência e Cultura. v.59, n.4, São Paulo, pp.2832, 2007.

SOUZA, R.D.F. de, et. al. Tecnologia industrial básica: histórico. In: Ministério da Ciência e Tecnologia, Confederação Nacional da Indústria, Serviço Nacional de Aprendizagem Industrial, Instituto Euvaldo Lodi. Tecnologia industrial básica; trajetória, tendências e desafios no Brasil. Brasília: MCT, CNI, SENAI/DN, IEL/NC, 2005. pp. 29-146.

TAN, K.C. A comparative study of 16 national quality awards. The TQM Magazine, v.14, n.3, 2002, pp. 165-171.

TAN, K.C. et. al. Factors affecting the development of national quality awards. Measuring Business Excelence, v.7, n.3, 2003, pp.37-45.

WINTER, S.G. Organizing for continuous improvement: evolucionary theory meets the quality revolution. In: BAUM, J.A.C., SINGH, J.V. (eds.). Evolutionary dynamics of organizations. New York: Oxford University Press, 1994, pp.90-108.

ZILBOVICIUS, M. Modelos para a produção, produção de modelos; gênese, lógica e difusão do modelo japonês de organização da produção. São Paulo: Fapesp - AnnaBlume, 1999.

\footnotetext{
${ }^{1}$ É justamente no início dos anos 1980 que o mundo da produção sofreu sério abalo, ao perceber que princípios e técnicas consagrados até então foram desafiados por uma lógica e um conjunto de práticas vindas do Japão.

2 As atividades de controle de qualidade se resumiam a inspeção, contagem, classificação e reparos. As rotinas (de busca) de solução de problemas estavam fora do campo de ação do departamento de inspeção (GARVIN, 1992). Além disso, tal departamento agia independentemente para alcançar seus objetivos. (COLE, 1999).

3 TUSHMAN, M.L., ANDERSON, P. Technological discontinuities and organizational environments. Administrative Science Quarterly, v.31, n.3, Sep.1986, pp.439-465.

${ }^{4}$ HARRISON, Michael I., SHIROM, A. Organizational diagnosis and assessment. Thousand Oaks: Sage, 1999.
} 

A introdução da concepção de excelência gerencial nos institutos e centros de pesquisa brasileiros - o projeto excelência na pesquisa tecnológica

${ }^{5}$ Hoje, além do Deming Prize, o Japão conta com o Japan Quality Award, que é uma premiação anual que reconhece a excelência da gestão da qualidade.

${ }^{6}$ Essas organizações atuaram na criação de padrões, identificação de gargalos, introdução de novas metodologias, treinamentos e disseminação de conhecimentos, dentre outras coisas. (COLE, 1999).

${ }^{7}$ O PBQP foi um Programa de governo, iniciado em 1990 com o objetivo de promover a qualidade e produtividade no Brasil, com vistas a aumentar a competitividade de bens e serviços produzidos no País.

${ }^{8}$ O gasto da China com P\&D em 2006 foi da ordem de US\$ 86.8 bilhões por ano, no período de 2001 a 2006 (OECD, 2008).

${ }^{9}$ Centro de Pesquisa e Desenvolvimento Leopoldo A. Miguez de Mello (CENPES), Fundação de Ciência e Tecnologia do RS (CIENTEC), Instituto Nacional de Tecnologia (INT), Instituto de Tecnologia do Paraná (TECPAR), Centro de Tecnologia Industrial Pedro Ribeiro (CETIND/SENAI), Fundação Centro de Referência em Tecnologias Inovadoras (CERTI), Centro de Pesquisa e Desenvolvimento (CEPED), Centro de Pesquisa de Energia Elétrica (CEPEL), Centro de Tecnologia Mineral (CETEM), Instituto de Pesquisa Tecnológica do Estado de São Paulo (IPT), Instituto de Tecnologia de Alimentos (ITAL), Centro de Desenvolvimento e Tecnologia Nuclear (CDTN), Fundação Núcleo de Tecnologia Industrial do Ceará (NUTEC), Instituto Brasileiro de Informação em Ciência e Tecnologia (IBICT), Instituto Brasileiro de Qualidade Nuclear (IBQN).

${ }^{10}$ O Projeto Excelência na Pesquisa Tecnológica havia dado grande visibilidade à ABIPTI nos últimos anos.

${ }^{11}$ No ano de 2008, o PEPT foi descontinuado e ressurgiu em junho de 2009. Nesse ano, 31 instituições aderiram àquele projeto (entre institutos de pesquisa, secretarias estaduais de C\&T e entidades associativas), mas apenas 05 participaram efetivamente do processo de avaliação (todos eles ICPs) (www.abipti.org.br).

${ }^{12}$ A revisão e adaptação dos CE-PNQ para o setor de pesquisa tecnológica jamais foram feitas, embora tenham sido propostas como trabalho de um bolsista sênior contratado para atuar no PEPT. 